\title{
OPTICALLY DETECTED CYCLOTRON RESONANCE STUDIES OF HIGH ELECTRON MOBILITY AlGaAs/GaAs STRUCTURES
}

\author{
M. GoDLEWSKI
}

Institute of Physics, Polish Academy of Sciences Al. Lotników 32/46, 02-668 Warszawa, Poland

\section{B. Monemar}

Dept. of Physics and Meas. Technol., Linköping Univ., 58183 Linköping, Sweden

\section{T.G. ANDERSON}

Dept. of Physics, Chalmers Univ. of Technol., Göteborg, Sweden

I. Tsimperidis, T. Gregorkiewicz, C.A.J. Ammerlaan

Van der Waals-Zeeman Lab., Univ. of Amsterdam, Amsterdam, The Netherlands

\section{J. Muszalski and M. KaniewsKa}

Institute of Electron Technology, Al. Lotników 32/46, 02-668 Warszawa, Poland

Optically detected cyclotron resonance is used for the identification of recombination transitions of two-dimensional electron gas in AlGaAs/GaAs heterostructures. Two photoluminescence emissions are attributed to the recombination of the two-dimensional electron gas. These are the so-called $\mathrm{H}$-band and the Fermi level singularity photoluminescence. Optical detection of cyclotron resonance is related to the change of the band bending across the GaAs active layer and the AlGaAs barrier, which is caused by impact ionization of shallow donors in the barrier region. Influence of a long range carrier scattering on ionized impurities on a mobility of the two-dimensional carriers is studied.

PACS numbers: 78.66.Fd, 73.40.Kp, 73.20.Dx, 71.25.Jd 


\section{Introduction}

Modulation doped high electron mobility (HEM) structures were introduced in order to separate spatially charge carriers from mobility limiting impurities [1]. In the $n$-type AlGaAs/GaAs HEM heterostructures electrons from AlGaAs donors are transferred to the GaAs layer, which results in their accumulation in a quasi-triangular potential on the GaAs side. Optical properties of such structures were first reported by Yuan et al. [2]. The so-called H-band photoluminescence (PL) emission was attributed to the radiative recombination of the two-dimensional (2D) electron gas accumulated at the AlGaAs/GaAs heterointerface with quasi-2D holes [3]. This attribution is confirmed by the present optically detected cyclotron resonance (ODCR) study.

For 2D electrons $z$ (perpendicular to heterointerface) motion of carriers is frozen by confining potential. Energy structure of such system with magnetic field normal to the heterointerface is made of a ladder of cyclotron levels, each level having singular DOS with degeneracy of $e B / h$. Cyclotron resonance (CR) is observed for magnetic field normal to heterointerface and no resonance of $2 \mathrm{D}$ carriers is found for field parallel to the heterointerface plane. Such angular anisotropy of the CR allows identification of recombination transitions of 2D carriers in the ODCR experiment [4].

\section{Experimental}

Three different $n$-type modulation doped $\mathrm{Al}_{x} \mathrm{Ga}_{1-x} \mathrm{As} / \mathrm{GaAs}$ heterostructures were studied. They were grown by the MBE method either in Göteborg (HEM 240) or in the Institute of Electron Technology, Warszawa (26.94 and 58.94). The HEM $240(x=0.35)$ heterostructure consisted of a $2000 \AA$ GaAs buffer, a 24 -period GaAs/AlAs short period $(20 \AA)$ superlattice, followed by a $500 \AA$ undoped GaAs active layer, a $200 \AA$ undoped AlGaAs spacer, an $800 \AA \mathrm{Si}$ doped $\left(10^{18} \mathrm{~cm}^{-3}\right) n$-type AlGaAs layer and finally a $50 \AA$ undoped GaAs cap layer. 26.94 and 58.94 HEM structures $(x=0.3)$ were grown on semi-insulating GaAs substrate followed by about $1 \mu \mathrm{m}$ undoped GaAs active layer, $160 \AA$ undoped AlGaAs spacer, $550 \AA \mathrm{Si}$ doped AlGaAs layer and $50 \AA$ thick Si doped $\left(1 \div 2 \times 10^{18} \mathrm{~cm}^{-3}\right) \mathrm{GaAs}$ cap layer. The 26.94 structure additionally contained $53 \AA$ thick Si doped GaAs layer and $1.68 \mu \mathrm{m}$ undoped GaAs layer on top of the $1 \mu \mathrm{m}$ GaAs layer.

In Fig. 1 we show PL spectra of three HEM structures studied. Two PL emission bands were observed in all three cases having different relative intensities and structure. The ODCR experiments were carried out for all three HEM structures with detailed study performed for the HEM 240 sample. In Fig. 2 we show electron CR detected via a decrease in the intensity (about $27 \%$ at $200 \mathrm{~mW}$ of microwave power) of the $8181 \AA$ PL emission. This CR signal overlapped heavily damped $\mathrm{CRs}$ of free electrons in AlGaAs barrier and GaAs substrate. A strong angular anisotropy (a) and power dependence of the width (b) were observed proving the $2 \mathrm{D}$ origin of the dominant CR signal. The spectral dependence of the ODCR signal was measured. CR of $2 \mathrm{D}$ electrons was observed via the decrease in the intensity of the first PL band with maximum at $8181 \AA$. This PL shifts from its initial position to about $8250 \AA$ for the $200 \mathrm{~mW}$ of the microwave power and its decrease 


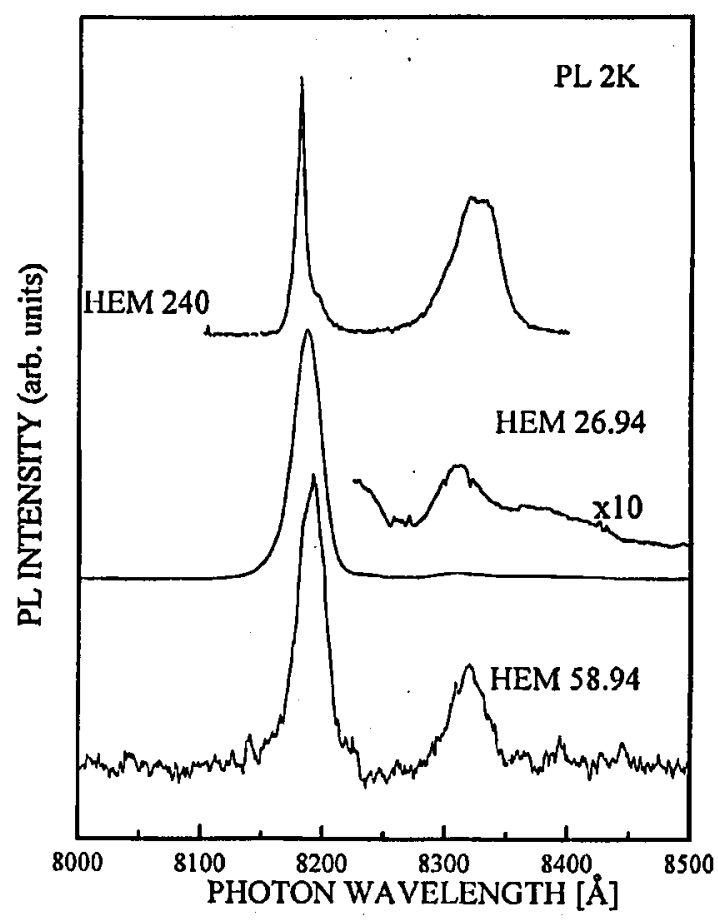

Fig. 1. Photoluminescence spectrum of the $n$-type modulation doped HEM structures measured at $2 \mathrm{~K}$.

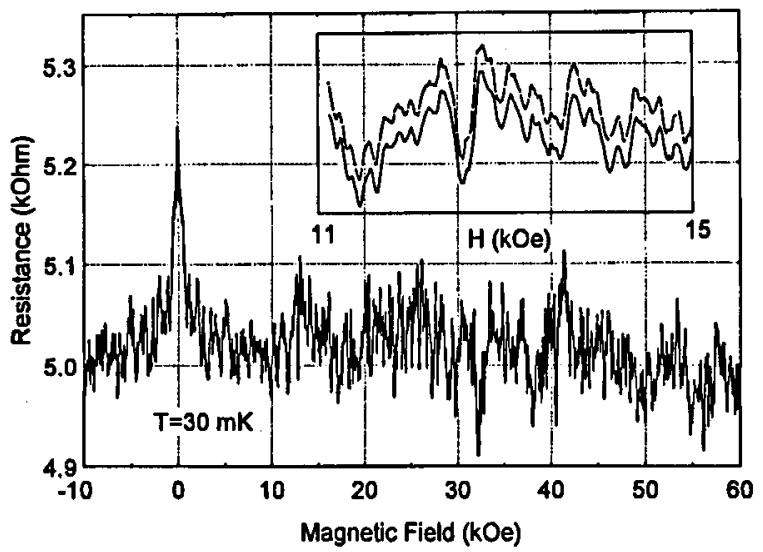

Fig. 2. Angular anisotropy (a) and microwave power dependence of the width (b) of the electron cyclotron resonance measured via the decrease in the intensity of the 2D-related PL of AlGaAs/GaAs HEM structure. CR observed consists of the relatively well resolved (for magnetic field normal to heterointerface, $0^{\circ}$ ) $2 \mathrm{D}$ electron resonance superimposed on heavily damped electron $\mathrm{CR}$ of $\mathrm{AlGaAs}$ barrier and GaAs substrate. 
in the intensity has a threshold dependence on the microwave power applied. We attribute the second PL band with maximum at $8325 \AA$ to the donor-acceptor pair emission of GaAs substrate and it will not be discussed here. The CR detected via this PL does not show any angular anisotropy confirming its 3D origin.

\section{Discussion}

The strong angular anisotropy of the CR signal detected via the first PL band allows us to attribute this PL emission to the recombination transitions of the 2D electron gas in the GaAs active layer of the HEM structures. The emission spectrum of 26.94 and 58.94 HEM structures consists of "typica". H-band PL. A slightly different PL shape was observed for the HEM 240 structure. The PL shows a pronounced peak at its high energy side. The electron-hole (e-h) interaction leading to exciton binding in HEM structures is reduced due to the weak screening of the Coulomb interaction and phase space filling effect and also the spatial electron-hole separation. Excitons are usually not observed for concentration of the 2D electron gas exceeding $3 \times 10^{11} \mathrm{~cm}^{-2}$ [5]. Electronic states due to $\mathrm{e}-\mathrm{h}$ correlation can, however, still exist for carriers at the Fermi level. We interpret the $8181 \AA$ emission of the AlGaAs/GaAs HEM 240 structure as such Fermi level singularity (FES) PL [6].

The intensity and spectral position of the H-band and FES PL emissions strongly depend on the experimental conditions. This reflects the PL dependence on the potential across the GaAs active layer [6]. The band bending across the HEM structure is reduced when photo-excited electrons neutralize ionized donors in the barrier and accumulate near the AlGaAs/GaAs interface [7]. The reduction of the band bending results in a spectral blue shift of the 2D-related PL emissions (towards a shorter wavelength) and increase in their intensity. By applying a positive gate voltage one can increase the localization of the $2 \mathrm{D}$ electrons at the $\mathrm{AlGaAs} / \mathrm{GaAs}$ heterointerfaces. In agreement with the recent calculations of Weegels et al. [7], the band bending across the GaAs active layer is then increased and the PL intensity is reduced. Similar effects we observed in the ODCR performed for increased microwa ve power, which allows us to conclude that the optical detection of the 2D electron $\mathrm{CR}$ is related to the change of the band bending across the GaAs active layer caused by imposed microwave power.

The ODCR investigations were performed under excitation with photon energy above the AlGaAs band gap, i.e., free carriers were photo-generated in the barrier which could neutralize ionized donors. This, together with an increase in 2D carrier density, reduces band bending across HEM structure and results in an increase in the intensity and spectral shift of the FES PL emission. Also the overlap between the wave functions of the $2 \mathrm{D}$ electrons and free holes is increased which further enhances the PL intensity. The threshold dependence on the microwave power reported above is a fingerprint of the impact ionization [4] of shallow donors in the AlGaAs barrier by microwave heated free carriers. The light induced neutralization of shallow donors in AlGaAs barrier is thus reduced in the ODCR experiment, which increases band bending across GaAs active layer and affects intensity and spectral position of the 2D-related PL. 
Concentration of ionized impurities in the barrier depends on the excitation intensity and microwave power applied, i.e., it varies depending on the experimental conditions. This is why the ODCR experiment allows us to verify the role of distant ionized impurities in scattering processes of $2 \mathrm{D}$ carriers. The microwave power dependence of $2 \mathrm{D}$ carriers mobility was estimated from experimental results shown in Fig. 2b. Mean scattering time of 2D electrons and their mobility was estimated from the CR width. The mobility varies from about $1.1 \times 10^{6} \mathrm{~cm}^{2} /(\mathrm{V} \mathrm{s})$ at low microwave power to $2 \times 10^{5} \mathrm{~cm}^{2} /(\mathrm{V} \mathrm{s})(\mathrm{HEM} 240)$ for $100 \mathrm{~mW}$ and $1 \times 10^{5} \mathrm{~cm}^{2} /(\mathrm{V} \mathrm{s})$ (HEM 58.94) for $200 \mathrm{~mW}$ microwave power. It is larger than the dark Hall mobility of our structures $\left(4 \times 10^{5} \mathrm{~cm}^{2} /(\mathrm{V} \mathrm{s})\right)$. The "dark" Hall mobility measured after illumination of the sample (after turning off the light) was increased, which partly can be explained by the correlation effects [8]. The decrease in the mobility observed in the ODCR experiment follows the threshold dependence of the PL intensity and position on the microwa ve power. This is why we relate the observed effect to the increased scattering of 2D electrons when the concentration of ionized impurities in the barrier is increased. The present study shows a pronounced influence of the above scattering mechanism on the low temperature mobility of the $2 \mathrm{D}$ electron gas.

This work was partly financed by the PBZ-101-01-11 program of the State Committee for Scientific Research (Republic of Poland).

\section{References}

[1] R. Dingle, H.L. Störmer, A.C. Gossard, W. Wiegmann, Appl. Phys. Lett. 33, 665 (1978).

[2] Y.R. Yuan, K. Mohammed, M.A.A. Pudensi, J.L. Merz, Appl. Phys. Lett. 45, 739 (1984).

[3] I.V. Kukushkin, K. von Klitzing, K. Ploog, Phys. Rev. B 45, 8509 (1988).

[4] M. Godlewski, W.M. Chen, B. Monemar, CRC Crit. Rev. Solid State Mater. Sci. 19, 241 (1994).

[5] D. Huang, H.Y. Chu, Y.C. Chang, R. Houdre, H. Morkoc, Phys. Rev. B 38, 1246 (1988).

[6] P.O. Holtz, B. Monemar, J.L. Merz, in: Semiconductor Interfaces and Microstructures, Ed. Zhe Chuan Feng, World Scientific, Singapore 1992, p. 93.

[7] L.M. Weegels, J.E.M. Haverkort, M.R. Leys, J.H. Wolter, Phys. Rev. B 46, 3886 (1992).

[8] T. Suski, P. Wiśniewski, I. Gorczyca, L.H. Dmowski, R. Piotrzkowski, P. Sobkowicz, J. Smoliner, E. Gornik, G. Böhm, G. Weimann, Phys. Rev. B 50, 2723 (1994). 